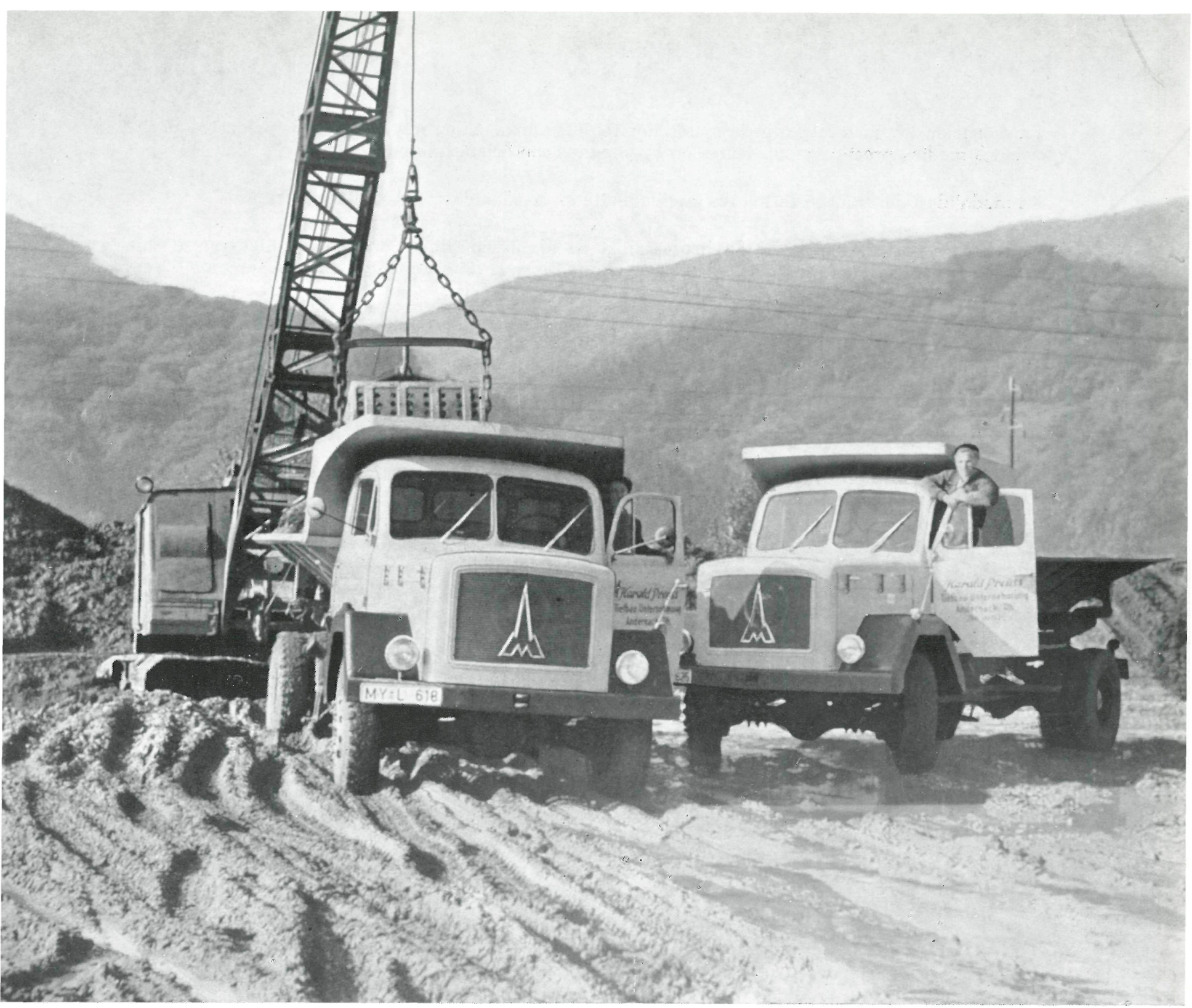

\title{
Famiomes y dumpers organización del transporte de materiales

\section{sinopsis}

La ejecución de un transporte de materiales requiere: una primera fase de adquisición de datos y una segunda de preparación de los medios y personal que han de intervenir en el trabajo.

La primera fase debe comprender: el reconocimiento y estudio de las zonas de carga y descarga, itinerarios, zonas de estacionamientos, medidas de seguridad, cálculo de necesidades y plan de transportes y circulación.

El cálculo de necesidades requiere la determinación práctica de la velocidad media de la unidad de transporte, a fin de clálar su de la maquinaria cargadora.

En el caso de trayecto variable, habrá que determinar la distancia crítica a partix de la cual será preciso introducir una nuecarga al no tener vehículo disponible.

En el caso de trayecto variable, habrá que determinar la distancia crítica a partir de la cual será preciso introducir una nueva unidad de transporte para que la máquina cargadora no esté insuficientemente servida.

Estas normas no son exactas, ya que la solución adoptada dependerá siempre de la flexibilidad de los medios disponibles y su utilización en un momento preciso, ya que al variar las circunstancias generales, en el transcurso de un mismo trabajo, no
podrá disponerse en todo momento de la solución más idónea. 
La ejecución de un transporte de materiales requiere una labor previa de preparación, es decir, colocar los medios precisos y suficientes en las mejores condiciones de realización.

La modalidad del transporte influye grandemente en su planteo y desarrollo.

La labor de preparación consta de dos fases: la de adquisición de los datos e informes que se estimen precisos para la confección del plan de movimiento, y la de aprestación de los vehículos y personal que han de intervenir en el trabajo.

La primera fase comprende los siguientes extremos:

Reconocimiento, estudio y designación de las zonas de carga y descarga.

Elección y designación de itinerarios.

Cálculo de necesidades.

Fijación de zonas de estacionamiento.

Medidas de seguridad a adoptar.

Plan de transportes y circulación.

El reconocimiento de las zonas de carga, descarga e itinerarios deberá comprender los siguientes puntos: Situación, clase y aptitud que presentan para la maniobra de vehículos y maquinaria auxiliar, superficie y estado del firme, limitaciones debidas a obras, pendientes, radios de giro mínimos, obstáculos e interferencias en el tráfico.

La fijación de zonas de estacionamiento estará condicionada a la situación de las mismas, posibilidades de accesos y salidas que ofrecen, dimensiones de las mismas y facilidades que presenten para la protección y abastecimiento del personal y vehículos.

Las medidas de seguridad serán todas aquéllas derivadas de la diversa maquinaria empleada.

El cálculo de necesidades trata de conseguir la determinación de un número de unidades de transporte con las que se consiga un equilibrio con la capacidad de la maquinaria de carga, de forma que el rendimiento económico sea óptimo. Está condicionado por las limitaciones inherentes a los medios disponibles y al entretenimiento de las pistas.

Los medios disponibles no serán, en general, los más apropiados en clase y número.

El entretenimiento de las pistas repercute intensamente en la posibilidad de conseguir promedios de velocidad elevados, con una importante disminución en los gastos de entretenimiento y conservación de los vehículos y un ahorro considerable de pérdidas sufridas por inmovilizaciones. Pese a que, en la generalidad de los casos, los gastos de ejecución y conservación de pistas son insignificantes en relación con la ganancia conseguida al obtener un funcionamiento más perfecto y eficiente de los medios disponibles, no suelen ser las pistas de transporte objeto de una primordial atención.

La velocidad media de un vehículo, dato fundamental para el cálculo de necesidades, no es, prescindiendo de los tiempos de carga y descarga, la media aritmética de las velocidades de ida y regreso. Para un trayecto de longitud 2L:

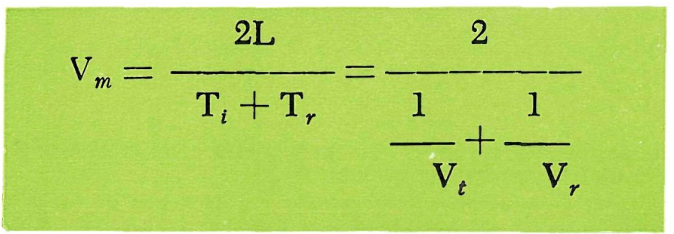

Si en un caso determinado un camión marcha cargado a una velocidad de $20 \mathrm{~km} / \mathrm{h}$, efectuando el regreso en vacío a una velocidad media de $50 \mathrm{~km} / \mathrm{h}$, siendo la distancia entre los centros de carga y descarga de $5 \mathrm{~km}$, la velocidad media no será:

$$
\frac{20+50}{2}=35 \mathrm{~km} / \mathrm{h}, \quad \text { sino: } \quad \frac{60 \times 10}{21}
$$

$21=28 \mathrm{~km} / \mathrm{h}$,


o bien:

$$
\frac{2}{\frac{1}{20}+\frac{1}{50}}=-\frac{200}{7}=28 \mathrm{~km} / \mathrm{h}
$$

Esta determinación de la velocidad media es tanto más importante cuanto más rápido sea el medio de transporte empleado, mayor la longitud de trayecto y más veces se efectúe éste.

Como las velocidades conseguidas dependen de imponderables, tales como intensidades del tráfico, efectividad de los conductores y otras circunstancias ocasionales, no será nunca exacta la velocidad determinada «a priori», valiendo únicamente como una primera aproximación; debiendo obtenerse, para poder fijar los datos reales a introducir en el cálculo los deducidos de valores prácticos obtenidos en el mayor número de viajes efectuados, siempre mediante la aplicación de la fórmula:

$$
\mathrm{V}_{m}=\frac{\text { número de trayectos efectuados }}{\frac{1}{\mathrm{~V}_{1}}+\frac{1}{\mathrm{~V}_{2}}+\frac{1}{\mathrm{~V}_{3}}+\ldots \frac{1}{\mathrm{~V}_{n}}}
$$

Del cálculo de la velocidad media se deduce el número de vehículos necesarios teniendo siempre en cuenta un factor de reserva, ya que normalmente será más conveniente tener un vehículo detenido en posición de espera que no inmovilizar la máquina cargadora, dada la relación de costos horarios de cada uno.

El procedimiento para determinar el número de unidades de transporte es distinto según se trate de trayectos fijos o de recorridos de longitud variable.

Para los casos de trayecto único puede emplearse la fórmula:

$$
\text { Número de unidades }=\frac{\text { Rendimiento de la máquina cargadora }}{\text { Rendimiento de la unidad de transporte }}
$$

Estos rendimientos se calcularán sobre la base de 60 min de eficiencia horaria para la máquina cargadora y $50 \mathrm{~min} / \mathrm{h}$ para el vehículo, con el fin de obtener un coeficiente de corrección de 60/50 que evite, en lo posible, las detenciones de la carga ante la falta de unidad de transporte disponible.

Habrá ocasiones, sin embargo, en que el trabajo de la máquina cargadora se desarrolle ininterrumpidamente en un lapso de varias horas de duración, en cuyo caso podrá presentarse la situación de no disponer de vehículo pese al coeficiente de seguridad introducido.

En los casos de trayecto de longitud variable, si la variación es continua y progresiva, habrá que determinar la distancia crítica a partir de la cual la máquina cargadora estará insuficientemente servida dada la nueva duración del ciclo y en la que habrá que introducir una nueva unidad.

Como en múltiples ocasiones, no será posible disponer en el momento preciso de la unidad de refuerzo, deberá considerarse previamente la pérdida que representa no disponer en el momento preciso de dicha unidad o el gasto que supone el mantenimiento de un vehículo innecesario, para la adopción de la solución más conveniente.

En todos los casos es prácticamente imposible lograr la solución óptima que más se adapte a las diversas situaciones que se irán presentando en el transcurso del trabajo, por lo que, de acuerdo con la flexibilidad de los vehículos que se posean, la mayor o menor facilidad de disponer de ellos en el momento preciso y los costes horarios de la diversa maquinaria deberán adoptarse soluciones, por exceso o por defecto, sirviendo únicamente los procedimientos exactos de cálculo como norma orientadora.

\section{Chinchilla}

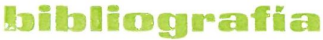

AdIL Gabay: Les Engins Mecaniques de Chantier. H. L. NICHoLs: Modern Techniques of excavation.
Teniente Coronel Mediavilla: Transportes Motorizados. NICHOLS, Jr.: How to operate excavation equipment. 


\section{Camions et dumpers}

\section{ORGANISATION DU TRANSPORT DE MATERIAUX}

La réalisation d'un transport de matériaux exige: une première phase pour l'obtention des données et une se. conde pour la préparation des moyens et du personnel qui doivent y parcitiper.

La première phase doit comprendre: la reconnaissance et l'étude des zones de charge et décharge, itinéraires, zones de stationnement, mesures de sécurité, calcul de nécessités et plan de transports et circulation.

Le calcul de nécessités demande la détermination pratique de la vitesse moyenne de l'unité de transport afin de calculer son rendement et d'obtenir l'équilibre optimum entre le nombre d'unités de transport à employer et la capacité des moyens de charge.

En cas de trajet unique il faudra introduire un facteur de sécurité pour éviter la paralysation des moyens de charge par manque de véhicules disponibles.

En cas de trajet variable, il faudra déterminer la distance critique, à partir de laquelle il conviendra d'introduire une nouvelle unité de transport pour que les moyens de charge ne soient pas insuffisamment desservis.

Cependant ces normes ne sont pas exactes. La solution adoptée dépendra toujours de la flexibilité des moyens disponibles et de leur utilisation dans un moment précis, car si les circonstances générales varient au cours d'un même travail, on ne pourra pas disposer à tout moment de la solution la plus indiquée.

\section{Treucks and dumperes}

\section{MATERIALS TRANSPORT ORGANISATION}

The transport of materials implies an initial phase of organisation, during which information is collected. This is followed by a second one, in which the personnel and equipment have to be prepared.

The first phase should include the inspection and study of the loading and unloading zones, the itinerary, the intermediate parking zones, security measures, estimate of overall needs and a circulation and transport plan. To estimate the material requirements it is necessary to determine the mean velocity of the transport units, and so calculate the average efficiency and the best compromise between the transport units to be employed and the capacity of the loading equipment.

If there is a single line of transport, a safety factor must be introduced to avoid total stoppage of the loading machinery if there is no available transport vehicle.

If the transport itinerary is variable, the critical distance will have to be worked out, such that for greater distances an additional transport unit should be added, to avoid the loading equipment being forced to work distances an additional tran
below its economical rate.

These recommendations are not rigid, since the solution to be adopted will always depend finally on the flexibility of the total means that are available for use at a given moment. As the general circumstances vary, in the course of a given project, the optimum method of handling a given difficulty will also vary.

\section{Lastrugenengen Dumper}

\section{ORGANISATION DES MATERIALTRANSPORTES}

Die Durchführung eines Materialtransportes erfordert eine erste Phase der Erlangung von Daten und eine zweite der Vorbereitung der Mittel und des Personals, die an der Arbeit teilzunehmen haben.

Die erste Phase soll die Untersuchung und das Studium der Zonen des Ladens und Abladens, der Wege, der Zonen des Parkens, der Sicherheitsmassnahmen, der Berechnung der Notwendigkeiten und des Transport- und Verkehrsplanes umfassen.

Die Berechnung der Notwendigkeiten erfordert die praktische Bestimmung der mittleren Geschwindigkeit der Transporteinheit, damit man ihren Ertrag berechnen kann und den besten Ausgleich unter der Anzahl der Trans porteinheiten anzuwenden erreicht und die Fähigkeit der Verlademaschinerie.

Im Falle der einzigen Strecke muss man einen Sicherheits- Faktor einführen, um die Stockung der Verlademaschinerie zu verhindern, wenn sie kein Fahrzeug zur Verfügung hat.

Im Falle der veränderbaren Strecke wird man die kritische Entfernung zu bestimmen haben, von der an man eine neue Transporteinheit einzuführen hat, damit die Verlademaschine nicht ungenügend bedient wird.

Diese Normen sind nicht genau, da die angenommene Lösung stets von der Biegsamkeit der verfügbaren Mittel und ihrer Benützung in einem notwendigen Augenblick abhängt, da man beim Aendern der allgemeinen Umstän de im Laufe derselben Arbeit nicht in jedem Augenblick über die geeigneteste Lösung wird verfügen können. 